\title{
BMJ Open Acupuncture for stable angina pectoris: a systematic review protocol
}

To cite: Yang M, Du T, Sun M, et al. Acupuncture for stable angina pectoris: a systematic review protocol. BMJ Open 2018;8:e019798. doi:10.1136/ bmjopen-2017-019798

- Prepublication history for this paper is available online. To view these files, please visit the journal online (http://dx.doi org/10.1136/bmjopen-2017019798).

MY and TD contributed equally.

Received 26 September 2017

Revised 18 January 2018

Accepted 8 February 2018

Check for updates

${ }^{1}$ School of Chinese Medicine, University of Hong Kong, Hong Kong

${ }^{2}$ Department of Rehabilitation, Xishan People's Hospital of Wuxi Municipality, Wuxi, China

${ }^{3}$ School of Acupuncture and Tuina, Chengdu University of TCM, Chengdu, China

${ }^{4}$ Department of Acupuncture and Rehabilitation, Teaching

Hospital of Chengdu University of Traditional Chinese Medicine, Chengdu, China

Correspondence to

Prof. Fanrong Liang;

acuresearch@126.com

\author{
Mingxiao Yang, ${ }^{1}$ Ting Du, ${ }^{2}$ Mingsheng Sun, ${ }^{3}$ Hulin Long, ${ }^{4}$ Dehua Li, ${ }^{4}$ Zhifu Shen, ${ }^{3}$ \\ Qiaofeng Wu, ${ }^{3}$ Lixing Lao, ${ }^{1}$ Fanrong Liang ${ }^{3}$
}

\begin{abstract}
Introduction Previous reviews indicate that the effect of acupuncture on stable angina pectoris (SAP) remains controversial. The results of trials published in the past 5 years may possibly change this situation, but an updated systematic review is not available. We therefore designed this study to systematically assess the efficacy and safety of acupuncture for treating SAP.
\end{abstract}

Methods and analysis Nine online databases will be searched without language or publication status restrictions from their inception to September 2017. Randomised controlled trials that include patients with stable angina receiving acupuncture therapy versus a control group will be deemed eligible. The selection of studies, data extraction and risk of bias assessment will be carried out by two independent reviewers. Data synthesis will be performed using RevMan V.5.3 software with either a fixed effects model or random effects model, depending on the heterogeneity test. Evidence quality will be evaluated using the Grading of Recommendations Assessment, Development and Evaluation system. The efficacy-effectiveness spectrum for each included trial will be rated using the Rating of Included Trials on the Efficacy-effectiveness Spectrum tool. Outcomes of interest include the improvement of weekly angina attacks and reduction of nitroglycerin medication use after receiving acupuncture treatment, the incidence of cardiovascular events, heart rate variability, pain intensity measured on a visual analogue scale, total workload and exercise duration at peak exercise, safety and adverse events. A metaanalysis will be conducted if no considerable heterogeneity is detected. The results will be presented as risk ratios with $95 \%$ Cls for dichotomous data and weighted mean differences or standardised mean differences with $95 \%$ Cls for continuous data.

Ethics and dissemination This systematic review will not involve private information from individuals or endanger their rights, and therefore does not necessarily require ethical approval. The results may be published in a peerreviewed journal or disseminated in relevant conferences. Trial registration number CRD42015016201.

\section{BACKGROUND}

Stable angina pectoris (SAP) is a prevalent cardiovascular disease that greatly compromises a patient's life quality and longevity. It is a clinical syndrome involving temporary hypoxic myocardial ischaemia and features sensations of burning, severe pain, pressure
Strengths and limitations of this study

Although systematic reviews of acupuncture for stable angina pectoris have been conducted previously, this study will update the evidence base by including many clinical trials that have been published in the past 5 years.

- Rating the efficacy-effectiveness spectrum of trials included in the systematic review has the potential to indicate how close current evidence is to 'real-world' practice.

- As several clinical outcomes have been used in published trials, a pooled analysis of all included studies may not be possible; however, subgroup analyses will be performed according to different outcomes.

and other forms of discomfort in the left anterior chest region. In the USA, SAP affects more than 7.8 million people and is reported to have an incidence of more than 0.5 million cases per year. ${ }^{1}$ In the European population, its incidence is estimated to be $10 \%-20 \%$ of people aged 65-74years. ${ }^{2}$ Within China, the prevalence is $2.4 \%$ among men and $3.2 \%$ among women, ${ }^{3}$ which makes it a serious public health problem. According to the 2013 European Society of Cardiology guidelines on the management of stable coronary artery disease, ${ }^{4}$ current treatment options for SAP are diverse, generally including lifestyle modification and elimination of risk factors, pharmacological management and revascularisation.

Although many therapeutic interventions are available to patients, the management of SAP is not satisfactory. Most patients continue to suffer from SAP or the side effects of their medications. ${ }^{5}$ Non-pharmacological therapies as secondary options are frequently chosen by patients. Acupuncture, a major component of traditional Chinese medicine, has been extensively used by clinicians for the management of acute attacks and for prophylaxis of SAP since ancient times. ${ }^{6} 7$ The clinical practice of acupuncture involves a series of procedures that entail the insertion of filiform needles into designated acupuncture 
points, followed by different needle manipulation techniques. Clinical observations and expert opinions suggest that acupuncture has the potential to reduce disease duration, angina attack frequency and nitroglycerin consumption, and improve cardiac work capacity. ${ }^{8-11}$ Studies suggest that acupuncture may exert a myocardial protection and vascular dilation effect against cardiac ischaemia and reperfusion by inhibiting the beta (1)-adrenoceptor signalling pathway and regulating myocardial enzyme action. ${ }^{12-17}$ Therefore, acupuncture has the potential to be an effective supplementary treatment option for SAP.

However, the effect of acupuncture for SAP is still controversial from the perspective of evidence-based medicine. Several systematic reviews have been conducted to assess the clinical benefits of acupuncture therapy for SAP, but all of them, including the most recent, which reviewed studies before 2013, were inconclusive due to poor-quality trials. ${ }^{18-20}$ Recently, it has been noticed with great interest that a rough estimation of at least 10 clinical studies has been published or conducted in the past 5 years. ${ }^{621-29}$ These studies have great potential to change the current evidence base of acupuncture for SAP. However, at present there exists no updated systematic review or study protocol published on this question. We thus have a unique opportunity to re-evaluate the issue and conceive this systematic review to determine the effectiveness and safety of acupuncture for patients with SAP based on the most comprehensive and up-to-date resources.

\section{METHODS}

\section{Criteria for including studies in the review}

Types of studies

The review will include randomised controlled trials (RCT) that were reported in any language. Completed and ongoing trials will be included. Trials using a two-arm or three-arm parallel design will be included. Crossover trials and quasi-RCTs will be excluded. Any study with a sample size of less than 10 participants will also be excluded from this review.

\section{Types of participants}

Trials including participants who meet the diagnostic criteria of SAP or stable coronary heart disease will be included. ${ }^{30-33}$ All eligible study participants will be included in this review regardless of their age, race or gender. Trials including study participants who are not appropriate to receive acupuncture therapy, such as pregnant or lactating women and those with additional severe diseases, will be excluded.

\section{Types of interventions}

The interventions considered in the studies must involve needle insertion at acupuncture points, pain points or trigger points, and be described as acupuncture. Adjunct therapy with acupuncture that is additive to the active treatment will be included in this review. However, other methods of stimulating acupuncture points without needle insertion (such as moxibustion, laser stimulation, massage or transcutaneous electrical nerve stimulation) will be excluded. Studies that compare the efficacy of different forms of acupuncture will be excluded as this is not the focus of the review.

\section{Types of comparator(s)/control}

The following control groups will be considered:

1. Acupuncture versus sham devices (interventions mimicking 'verum' acupuncture/treatment, but deviating in at least one aspect considered important by acupuncture theory, such as skin penetration or non-acupoint location).

2. Acupuncture versus routine care.

3. Acupuncture versus conventional drugs.

4. Acupuncture in addition to active treatment versus active treatment alone.

Studies that only compare different forms of acupuncture or compare acupuncture with other complementary and alternative therapeutic interventions shall be excluded.

\section{Types of outcome measures}

\section{Primary outcomes}

The number of weekly angina attacks and nitroglycerin use during a period of at least 2 weeks following randomisation.

\section{Secondary outcomes}

1. Cardiovascular events.

2. ECG changes (mainly ST-segment depression).

3. Heart rate variability.

4. Angina pain intensity (assessed by visual analogue scale).

5. Affective emotions (assessed by self-rating anxiety scale and self-rating depression scale).

6. Total workload (in Met-minutes) and exercise duration (in seconds) at peak exercise.

7. Overall tolerability. ${ }^{34}$

8. Overall acceptability. ${ }^{34}$

\section{Search methods for identification of studies} Electronic searches

The following databases will be searched from their inception to September 2017: MEDLINE, Ovid, EMBASE, Cochrane Library, the Allied and Complementary Medicine Database (AMED), Chinese National Knowledge Infrastructure (CNKI), Chinese Biomedical Literature Database (CBM), Wanfang Database, the Chongqing VIP Chinese Science and Technology Periodical Database (VIP). Only RCTs that evaluate the effect of acupuncture in comparison with the aforementioned comparator controls will be included. There will be no language restriction on the included trials. The following medical search headings $(\mathrm{MeSH})$ will be used: stable angina pectoris, stable angina, angina pectoris, angina, acupuncture, acupuncture therapy, electroacupuncture, electroacupuncture therapy, manual acupuncture, acupoint, 


\begin{tabular}{|c|c|}
\hline$\# 1$ & randomized controlled trial [pt] \\
\hline \#2 & controlled clinical trial [pt] \\
\hline \#3 & randomized [tiab] \\
\hline \#4 & placebo [tiab] \\
\hline \#5 & clinical trials as topic [mesh: noexp] \\
\hline \#6 & randomly [tiab] \\
\hline \#7 & trial [ti] \\
\hline \#8 & \#1 OR \#2 OR \#3 OR \#4 OR \#5 OR \#6 OR \#7 \\
\hline \#9 & animals [mh] NOT humans [mh] \\
\hline \#10 & \#8 NOT \#9 \\
\hline \#11 & angina, stable [mesh] \\
\hline \#12 & chronic stable angina pectoris [tiab] \\
\hline \#13 & stable angina pectoris [tiab] \\
\hline \#14 & chronic stable angina [tiab] \\
\hline \#15 & stable angina [tiab] \\
\hline \#16 & angina pectoris [ti] \\
\hline \#17 & angina [ti] \\
\hline \#18 & \#11 OR \#12 OR \#13 OR \#14 OR \#15 OR \#16 OR \#17 \\
\hline \#19 & acupuncture [mesh] \\
\hline \#20 & acupuncture therapy [mesh] \\
\hline \#21 & electroacupuncture [tiab] \\
\hline \#22 & electroacupuncture therapy [tiab] \\
\hline \#23 & manual acupuncture [tiab] \\
\hline \#24 & dry needle [tiab] \\
\hline \#25 & acupoint [tiab] \\
\hline \#26 & \#19 OR \#20 OR \#21 OR \#22 OR \#23 OR \#24 OR \#25 \\
\hline \#27 & \#10 AND \#18 AND \#26 \\
\hline \#28 & remove duplicates from \#27 \\
\hline
\end{tabular}

randomised controlled trial, randomised controlled, randomised, controlled, clinical trial, comparative study, prospective study. Chinese translations of these search terms will be used for the Chinese databases. The searching strategy for MEDLINE is listed in table 1. The search strategy will be modified according to the Cochrane Handbook for Systematic Reviews ${ }^{35}$ for other databases.

\section{Searching other resources}

Ongoing trials with unpublished data will be retrieved from the following clinical trial registries: the NIH clinical registry ClinicalTrials.gov (https://www.clinicaltrials. gov/), the International Clinical Trials Registry Platform (ICTRP) (http://www.who.int/ictrp/en/), the Australian New Zealand Clinical Trials Registry (http://www. anzctr.org.au/) and the Chinese clinical registry (http:// www.chictr.org/en/). The list of all identified publications including relevant systematic reviews and meta-analyses will be reviewed to further identify additional trials.
Useful but incomplete data will be obtained for data synthesis from the contact trial personnel.

\section{Data collection and analysis \\ Selection of studies}

The search results will be imported from the original databases to Endnote V.X7.1. Two reviewers (MY and MS) will independently assess the eligibility of the retrieved studies according to the inclusion criteria. For preliminary study selection, only the title and abstract will be reviewed to exclude obviously inappropriate publications. Unmatched studies will be removed to a trash box in the software. The reasons for exclusion will be recorded as an Excel data set. The next step will be to further evaluate the included studies by reading their full-text version. The reference list will be checked by the two reviewers to identify potentially missing trials. The selection results will be cross-checked by the two reviewers. Any disagreement will be resolved by consensus. Further argument will be arbitrated by a third reviewer (TD). Each eligible trial will be assigned a study ID formatted as follows: surname of the first author + space + year of publication (eg, Yang 2017).

\section{Data extraction and management}

Two reviewers (HL and ZS) will independently doublecheck the eligibility of the included studies and extract data by entering details into a predefined data acquisition form. This acquisition form will include four main domains: citation information (title, author list, source of publication, year of publication, first author's name and affiliation, country, sponsor), design (design, participants, trial methods, duration, intervention details, caregiver information), results (outcome measures, adverse events) and conclusion. Any discrepancy noticed in the process of data cross-checking will be resolved through discussion and the suggestion of a third reviewer (LL).

Assessment of risk of bias in included studies

The risk of bias for each included trial will be evaluated using the Cochrane Collaboration's tool for assessing risk of bias in randomised trials. ${ }^{36}$ Two reviewers (MY and TD) will input the relevant details of each trial into the RevMan software $^{37}$ (V.5.3) and assess the trial for at least six domains (random sequence generation, allocation concealment, blinding of participants and personnel, blinding of outcome assessment, incomplete outcome data, selective reporting and other bias if necessary). For each domain, the trial will be rated as high, unclear or low risk of bias. A trial that is rated high risk of bias in one or more domains will be rated as 'high risk', while a low risk of bias in all domains will be rated as 'low risk'. If there is low or unclear risk of bias for all key domains, the trial will be rated as 'unclear risk'. ${ }^{36}$ The contact person or corresponding author will be contacted if basic information is missing for the risk of bias assessment. The rating results will be cross-checked and discrepancies resolved through discussions and the arbitration of a third reviewer (FL). 
Measures of treatment effect

Efficacy data will be synthesised and statistically analysed in RevMan V.5.3. Dichotomous data will be analysed by using a risk ratio with $95 \%$ CIs. For continuous outcomes, data will be analysed by using a weighted mean difference (WMD) or a standard mean difference (SMD) with $95 \%$ CIs. The WMD will be used for the same scale or same assessment instrument; SMD will be used for different assessment tools.

\section{Unit of analysis issues}

The units of each outcome from different trials will be converted to the International System of Units before statistical analysis.

\section{Dealing with missing data}

Whether the data were missing on purpose or 'at random' will be predetermined by contacting the corresponding author or relevant author. Available data will be analysed following confirmation by investigators that the data were missing 'at random'. ${ }^{35}$ If not, a request for missing data will be sent to the original investigators of the trial, or the contact person recorded in the trial registry. In case of no reply from the authors or contact person, we will impute the missing data with replacement values, treating these as if they were observed. The last observation carried forward imputation method will be used to assume a missing value and then an intention-totreat analysis will be performed. Moreover, if possible, we will perform sensitivity analyses to assess how sensitive the results are to reasonable changes in the assumptions that are made. The potential impact of the effect of missing data on the final findings of the review will be addressed in the discussion.

\section{Assessment of heterogeneity}

$\mathrm{X}^{2}$ tests will be performed in the forest plot using RevMan V.5.3 to investigate the statistical heterogeneity and a $p$ value of less than 0.10 will be considered significant, in line with the Cochrane Handbook. ${ }^{35}$ Moreover, the $\mathrm{I}^{2}$ value will be calculated to quantify the impact of the statistical heterogeneity on the meta-analysis. The Cochrane Handbook classifies the $\mathrm{I}^{2}$ values into four categories: $0 \%-40 \%$, might not be important; $30 \%-60 \%$, indicates moderate heterogeneity; $50 \%-90 \%$, represents substantial heterogeneity; $75 \%-100 \%$, suggests considerable heterogeneity.

\section{Assessment of reporting biases}

A funnel plot will be generated to observe the reporting bias when more than 10 trials are included. ${ }^{35}$

\section{Data synthesis}

Clinical data will be imported into RevMan software (V.5.3) to perform data synthesis. Data will be synthesised and analysed depending on the level of statistical heterogeneity. If the heterogeneity tests show little or no statistical heterogeneity in these trials, the fixed effects model will be used for the pooled data. If significant heterogeneity is detected (if the $\mathrm{I}^{2}$ value is no less than $50 \%$ ), the random effects model will be used for data synthesis. If there is considerable heterogeneity in the trials, meta-analysis will not be performed. In this case, we will try to identify the source of heterogeneity from both clinical and methodological aspects and a narrative, qualitative summary will be provided.

\section{Subgroup analysis and investigation of heterogeneity}

If data are available, a subgroup analysis will be conducted according to variations in the characteristics of the trial participants and acupuncture treatments. When considerable heterogeneity is detected in a previous analysis, a subgroup analysis will be performed if necessary.

\section{Sensitivity analysis}

Sensitivity analysis will be used to monitor the robustness of the primary decision made in the review process. We will consider several decision nodes within the process of the systematic review to implement a sensitivity review, such as small studies, methodological weaknesses and missing data. The sensitivity analysis will involve two steps, as suggested by the Cochrane Handbook: first, including all studies as the primary meta-analysis does and second, including those that are definitely known to be eligible. The results of the sensitivity analysis will be presented in summary tables. The risk of bias in the review process, as indicated by the results of the sensitivity analysis, will be discussed.

\section{Other analysis}

Consideration will be given to using a metaregression analysis to investigate the impact of the year of publication on the outcome estimates.

\section{Evidence quality evaluation}

The reviewers will use the online Grading of Recommendations Assessment, Development and Evaluation (GRADE) application, GRADEpro (https://gradepro. $\mathrm{org} /$ ), to independently assess the quality of evidence for each outcome. ${ }^{38}$ Evidence quality will be rated 'high', 'moderate', 'low' or 'very low' according to the GRADE rating standards. ${ }^{39} 40$ The quality of evidence of a specific study will be assessed according to the appropriateness of the study design, soundness of implementation, directness and precision of evidence, consistency or homogeneity of the results and other biases. A summary of findings table will be generated and included in the final report.

\section{Efficacy-effectiveness spectrum analysis}

Because systematic reviews can include both explanatory trials and pragmatic trials for data synthesis, it is important for clinicians and researchers to know the nature of the evidence. Therefore, to analyse the efficacy-effectiveness spectrum of each included trial, the Rating of Included Trials on the Efficacy-effectiveness Spectrum scale ${ }^{41}$ will be used. Four domains will be assessed to justify the efficacy-effectiveness spectrum for each trial: participant 
characteristics, trial setting, flexibility of interventions and clinical relevance of interventions.

\section{Ethics and dissemination}

This review will not involve private information from individuals or compromise their rights, and therefore does not require ethical approval. The results may be published in a peer-reviewed journal or disseminated at relevant conferences. Due to the paucity of related publications in the field, this review article will, by adding more recent studies into the analysis, provide more robust evidence of acupuncture therapy for treating stable angina, and lead to informed clinical practice and acupuncture research.

Acknowledgements We thank Professor Christopher Zaslawski from the University of Technology, Sydney (Australia) for copy-editing the manuscript. We thank Professor Ji Chen from the Chengdu University of Traditional Chinese Medicine for revising the draft of the manuscript.

Contributors MY conceived the review protocol and drafted the manuscript. LL, $\mathrm{QW}, \mathrm{DL}$ and FL revised the study design. TD, MS, FL and HL participated in the design of the search strategy and data extraction data set. MY, TD, MS and ZS formed the data synthesis and analysis plan. In practice, FL and LL will monitor each procedure of the review and are responsible for the quality control. All authors have read and approved the publication of the protocol.

Funding This work is supported by a grant from the National Natural Science Foundation of China (No. 81590951, 81403482), and a grant from the Ministry of Science and Technology of China, the National Key Basic Research Program for Development (973 Program) (grant number 2012CB518501).

Disclaimer The sponsors have no role in design, conducting or revising the study protocol.

Competing interests None declared.

Patient consent Not required.

Provenance and peer review Not commissioned; externally peer reviewed.

Open Access This is an Open Access article distributed in accordance with the Creative Commons Attribution Non Commercial (CC BY-NC 4.0) license, which permits others to distribute, remix, adapt, build upon this work non-commercially, and license their derivative works on different terms, provided the original work is properly cited and the use is non-commercial. See: http://creativecommons.org/ licenses/by-nc/4.0/

(c) Article author(s) (or their employer(s) unless otherwise stated in the text of the article) 2018. All rights reserved. No commercial use is permitted unless otherwise expressly granted.

\section{REFERENCES}

1. Go AS, Mozaffarian D, Roger VL, et al. Executive summary: heart disease and stroke statistics--2013 update: a report from the American Heart Association. Circulation 2013;127:143-6.

2. Cardiology TFotMoSAPotESo. Guidelines on the management of stable angina pectoris. Eur Heart J 2006;27:1341-81.

3. Lam TH, Liu LJ, Janus ED, et al. Fibrinogen, angina and coronary heart disease in a Chinese population. Atherosclerosis 2000;149:443-9.

4. Montalescot G, Sechtem U, Achenbach S, et al. 2013 ESC guidelines on the management of stable coronary artery disease: the Task Force on the management of stable coronary artery disease of the European Society of Cardiology. Eur Heart J 2013;34:2949-3003.

5. Tarkin JM, Kaski JC. Pharmacological treatment of chronic stable angina pectoris. Clin Med 2013;13:63-70.

6. $\mathrm{Xu} \mathrm{L}, \mathrm{Xu} \mathrm{H}, \mathrm{Gao} W$, et al. Treating angina pectoris by acupuncture therapy. Acupunct Electrother Res 2013;38:17-35.

7. Kang X, Xia Y. Acupuncture on arrhythmia and other cardiac diseases. Acupuncture therapy for neurological diseases: Springer, 2010:263-88.
8. Ballegaard S, Meyer CN, Trojaborg W. Acupuncture in angina pectoris: does acupuncture have a specific effect? J Intern Med 1991;229:357-62.

9. Ballegaard S, Pedersen F, Pietersen A, et al. Effects of acupuncture in moderate, stable angina pectoris: a controlled study. J Intern Med 1990;227:25-30.

10. Ballegaard S, Jensen G, Pedersen F, et al. Acupuncture in severe, stable angina pectoris: a randomized trial. Acta Med Scand 1986;220:307-13.

11. Yang G, Li C, Wang R, et al. A summary of prof. Wang Rui's clinical experience on using acupoints to treat cardiovascular diseases. Clinical Journal of Acupuncture and Moxibustion 2016;6:59-60.

12. Zhang H, Liu L, Huang G, et al. Protective effect of electroacupuncture at the Neiguan point in a rabbit model of myocardial ischemia-reperfusion injury. Can J Cardiol 2009;25:359-63.

13. Yang L, Yang J, Wang Q, et al. Cardioprotective effects of electroacupuncture pretreatment on patients undergoing heart valve replacement surgery: a randomized controlled trial. Ann Thorac Surg 2010;89:781-6.

14. Gao J, Fu W, Jin Z, et al. Acupuncture pretreatment protects heart from injury in rats with myocardial ischemia and reperfusion via inhibition of the beta(1)-adrenoceptor signaling pathway. Life Sci 2007;80:1484-9.

15. Zhou W, Ko Y, Benharash P, et al. Cardioprotection of electroacupuncture against myocardial ischemia-reperfusion injury by modulation of cardiac norepinephrine release. Am J Physiol Heart Circ Physiol 2012;302:H1818-H1825.

16. Huang R, Han S, Qin L, et al. Effect of electroacupuncture pretreatment on myocardial enzyme levels in recurrent myocardial ischemia rabbits]. Zhen ci yan jiu= Acupuncture research/[Zhongguo yi xue ke xue yuan Yi xue qing bao yan jiu suo bian ji] 2012;37:224-8.

17. Shen J. Research on the neurophysiological mechanisms of acupuncture: review of selected studies and methodological issues. $J$ Altern Complement Med 2001;7(Suppl 1):121-7.

18. Zhang Z, Chen M, Zhang L, et al. Meta-analysis of acupuncture therapy for the treatment of stable angina pectoris. Int J Clin Exp Med 2015;8:5112.

19. Yu C, Ji K, Cao H, et al. Effectiveness of acupuncture for angina pectoris: a systematic review of randomized controlled trials. BMC Complement Altern Med 2015:15:90.

20. Chen J, Ren $\mathrm{Y}$, Tang $\mathrm{Y}$, et al. Acupuncture therapy for angina pectoris: a systematic review. J Tradit Chin Med 2012;32:494-501.

21. Wang M, Chen H, Lu S, et al. [Impacts on neutrophil to lymphocyte ratio in patients of chronic stable angina pectoris treated with acupuncture at Neiguan (PC 6)]. Zhongguo Zhen Jiu 2015;35:417-21.

22. Wang N, Lu SF, Chen $\mathrm{H}$, et al. A protocol of histone modificationbased mechanistic study of acupuncture in patients with stable angina pectoris. BMC Complement Altern Med 2015;15:139.

23. Ren Y, Li D, Zheng H, et al. Acupoint application in patients with chronic stable angina pectoris: study protocol of a randomized, double-blind, controlled trial. Evid Based Complement Alternat Med 2014;2014:1-8.

24. Li D, Yang M, Zhao L, et al. Acupuncture for chronic, stable angina pectoris and an investigation of the characteristics of acupoint specificity: study protocol for a multicenter randomized controlled trial. Trials 2014;15:50.

25. Mehta PK, Polk DM, Zhang X, et al. A randomized controlled trial of acupuncture in stable ischemic heart disease patients. Int $J$ Cardiol 2014;176:367-74.

26. Li YC, Guo L. [Thirty-five cases of stable and exertional angina pectoris treated with acupuncture of supplementing and activating Zongqi]. Zhongguo Zhen Jiu 2013;33:173.

27. Zhang Z, Zhang F, Wang Y, et al. Traditional Chinese medicine for stable angina pectoris via TCM pattern differentiation and TCM mechanism: study protocol of a randomized controlled trial. Trials 2014; $15: 422$.

28. Li X, Cao J, Jiang A. [Clinical observation and dynamic electrocardiogram of unstable angina pectoris treated with acupuncture combined with western medication]. Zhongguo Zhen Jiu 2015;35:895.

29. Liao G, Lan J, Zhang X. The effect of Wu You Time point application therapy on the quality of life in patients with coronary heart disease angina pectoris [J]. Nursing Practice and Research 2012;16:004.

30. Fraker TD, Finn SD, Gibbons RJ, et al. 2007 chronic angina focused update of the ACC/AHA 2002 guidelines for the management of patients with chronic stable angina: a report of the American College of Cardiology/American Heart Association Task Force on Practice Guidelines Writing Group to develop the focused update of the 2002 guidelines for the management of patients with chronic stable angina. J Am Coll Cardiol 2007;50:2264-74. 
31. Gibbons RJ, Abrams J, Chatterjee K, et al. ACC/AHA 2002 guideline update for the management of patients with chronic stable angina-summary article: a report of the American College of Cardiology/ American Heart Association Task Force on practice guidelines (Committee on the Management of Patients With Chronic Stable Angina). J Am Coll Cardiol 2003;41:159-68.

32. Fox K, Garcia MA, Ardissino D, et al. Guidelines on the management of stable angina pectoris: executive summary: The Task Force on the Management of Stable Angina Pectoris of the European Society of Cardiology. Eur Heart J 2006;27:1341-81.

33. Chinese Society of Cardiology, Chinese Medical AssociationEditorial Board, Chinese Journal of Cardiology. [Guideline for diagnosis and treatment of patients with chronic stable angina (no abstract)]. Zhonghua Xin Xue Guan Bing Za Zhi 2007;35:195-206.

34. Zhou X, Qin B, Whittington C, et al. Comparative efficacy and tolerability of first-generation and newer-generation antidepressant medications for depressive disorders in children and adolescents: study protocol for a systematic review and network meta-analysis. BMJ Open 2015;5:e007768.
35. Higgins JP, Green S. Cochrane handbook for systematic reviews of interventions: Wiley Online Library, 2008.

36. Higgins JP, Altman DG, Gøtzsche PC, et al. The Cochrane Collaboration's tool for assessing risk of bias in randomised trials. BMJ 2011;343:d5928.

37. Collaboration RTC. Review Manager (RevMan). 5.0. Copenhagen: The Nordic Cochrane Collaboration, 2008.

38. Guyatt GH, Oxman AD, Vist GE, et al. GRADE: an emerging consensus on rating quality of evidence and strength of recommendations. BMJ 2008;336:924-6.

39. Guyatt GH, Oxman AD, Vist GE, et al. Rating quality of evidence and strength of recommendations: GRADE: an emerging consensus on rating quality of evidence and strength of recommendations. BMJ: British Medical Journal 2008;336:924.

40. Guyatt $\mathrm{GH}$, Oxman $\mathrm{AD}$, Schünemann $\mathrm{HJ}$, et al. GRADE guidelines: a new series of articles in the Journal of Clinical Epidemiology. J Clin Epidemiol 2011;64:380-2.

41. Wieland LS, Berman BM, Altman DG, et al. Rating of Included Trials on the Efficacy-Effectiveness Spectrum: development of a new tool for systematic reviews. J Clin Epidemiol 2017;84:95-104. 